
CONF-961113-23
SAN096-2010C

Properties of Small-Aperture Selectively Oxidized VCSELs

Kent D. Choquette, W. W. Chow, G. Ronald Hadley, H. Q. Hou, and K. M. Geib

Photonics Research Department

Sandia National Laboratories

Albuquerque, NM 87185

\section{RECEIVED \\ AUG 151996 \\ OSTI}

\begin{abstract}
We report an analysis of the size dependence of VCSEL threshold which agrees with experimental results. The increasing threshold current density of small area VCSELs arises from both increasing threshold gain and increasing leakage current.
\end{abstract}

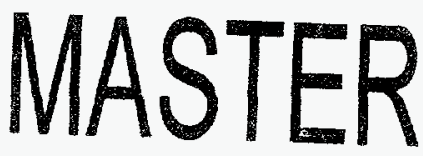

DISTREMTON OF THS DOCIMENT IS INMMTED 'P 


\section{DISClAIMER}

Portions of this document may be illegible in electronic image products. Images are produced from the best available original document. 


\title{
Properties of Small-Aperture Selectively Oxidized VCSELs
}

\author{
Kent D. Choquette, W. W. Chow, G. Ronald Hadley, H. Q. Hou, and K. M. Geib \\ Center for Compound Semiconductor Technology \\ Sandia National Laboratories \\ Albuquerque, NM 87185 \\ (505)844-7287 kdchoqu@ sandia.gov
}

Using selective oxidation of AlGaAs layers to form oxide apertures within vertical-cavity surface emitting lasers (VCSELs) has enabled dramatic decreases in threshold current as a result of lower absorption[1], increased spontaneous emission coupling[2], and more efficient optical[3] and electrical[4] confinement in extremely small active volumes. However, optical scattering from oxide apertures has been shown to increase with decreasing laser size[5], although without good agreement between present models and experiment[6]. We report an accurate analysis of the size dependence of the threshold properties of selectively oxidized VCSELs. Unique electronic and optical models have been combined to investigate the performance of our present VCSEL design. The increasing threshold current density, $\mathrm{J}_{\mathrm{th}}$, for small lasers is found to arise from the higher threshold gain required to overcome aperture optical scattering and from increasing leakage current present at the high carrier densities. The agreement between our analysis and experiment validates our models and provides insight for the design of ultralow threshold VCSELs.

Fig. 1 shows the monolithic selectively oxidized $850 \mathrm{~nm}$ VCSEL with a 21 period top mirror. Single or five buried oxide layers are positioned on one or both sides of the optical cavity to confine the current into the 5 quantum well active region. VCSELs with cross section areas varying from $\approx 0.5$ to $1000 \mu \mathrm{m}^{2}$ are examined. In Fig. 2 we plot $J_{\text {th }}$ versus size and find for apertures $<100 \mu \mathrm{m}^{2}, \mathrm{~J}_{\mathrm{th}}$ increases significantly due to optical scattering from the apertures, which becomes more severe using multiple apertures and/or apertures on both side of the cavity.

Fig. 3 shows the threshold material threshold gain versus device size for the VCSELs with a single aperture on each side of the optical cavity. The points in Fig. 3 are determined from relating the measured intrinsic threshold voltage of the VCSELs to the calculated quasi-Fermi level splitting due a given carrier density in the quantum well structure producing gain[7]. The ohmic drop arising from the mirror series resistance is subtracted from the measured VCSEL threshold voltage to determine the intrinsic voltage for the gain calculation. The gain calculation incorporates band structure and many-body contributions to the laser gain[8]. The solid curve in Fig. 3 results from a first-principles 2-dimensional finite difference calculation of the optical modes within the VCSEL and accurately takes into account the optical scattering from the oxide apertures[9]. The good agreement between these calculations serves to validate our optical and gain models and corroborates the increase of threshold gain due to increasing optical loss for small VCSEL cross section area. Note the high threshold gain that is predicted for the smallest VCSELs is consistent with the absence of lasing observed for the smallest $\left(<2 \mu \mathrm{m}^{2}\right)$ devices examined.

In Fig. 4 we compare the measured and calculated $\mathrm{J}_{\text {th }}$ and the calculated leakage current for VCSELs with a single aperture on each side of the optical cavity. The leakage current is determined by assuming thermal equilibrium between confined and unconfined populations of the quantum well structure, where the latter contribute to leakage[7]. Fig. 4 demonstrates that the leakage current also increases with decreasing VCSEL size due to high threshold carrier density. Thus the increase of $\mathrm{J}_{\mathrm{th}}$ arises from both increasing optical loss and increasing leakage current.

In summary, we have developed an accurate first-principles analysis to probe the threshold properties of selectively oxidized VCSELs. The analysis of our present VCSELs indicates that in order to achieve ultralow threshold, oxide aperture scattering loss and leakage currents must be addressed. The agreement between our calculations and experiment solidify our understanding and enable the identification of fundamental limitations of low threshold VCSEL operation. The performance and analysis of modified VCSEL designs will be reported.

This work is supported by the U.S. DOE under contract DE-AC04-94AL85000. 
[1] G. M. Yang, et al., Electron. Lett. 31, 886 (1995).

[2] D. G. Deppe, et al., Photon Tech Lett. 7, 965 (1995).

[3] K. L. Lear, et al., Appl. Phys. Lett. 66, 2616 (1995).

[4] K. D. Choquette, et al., Appl. Phys. Lett. 66, 3413 (1995).

[5] K. L. Lear, et al., 1995 LEOS Annual Meeting, paper SCL 16.7 (1995).

[6] B. J. Thibeault et al., Photon Tech Lett. 8, 593 (1996).

[7] K. D. Choquette, et al., Appl. Phys. Lett. 68 (1996).

[8] W. Chow, et al., Semiconductor Laser Physics, Springer-Verlag (Berlin, 1994).

[9] G. R. Hadley, unpublished.
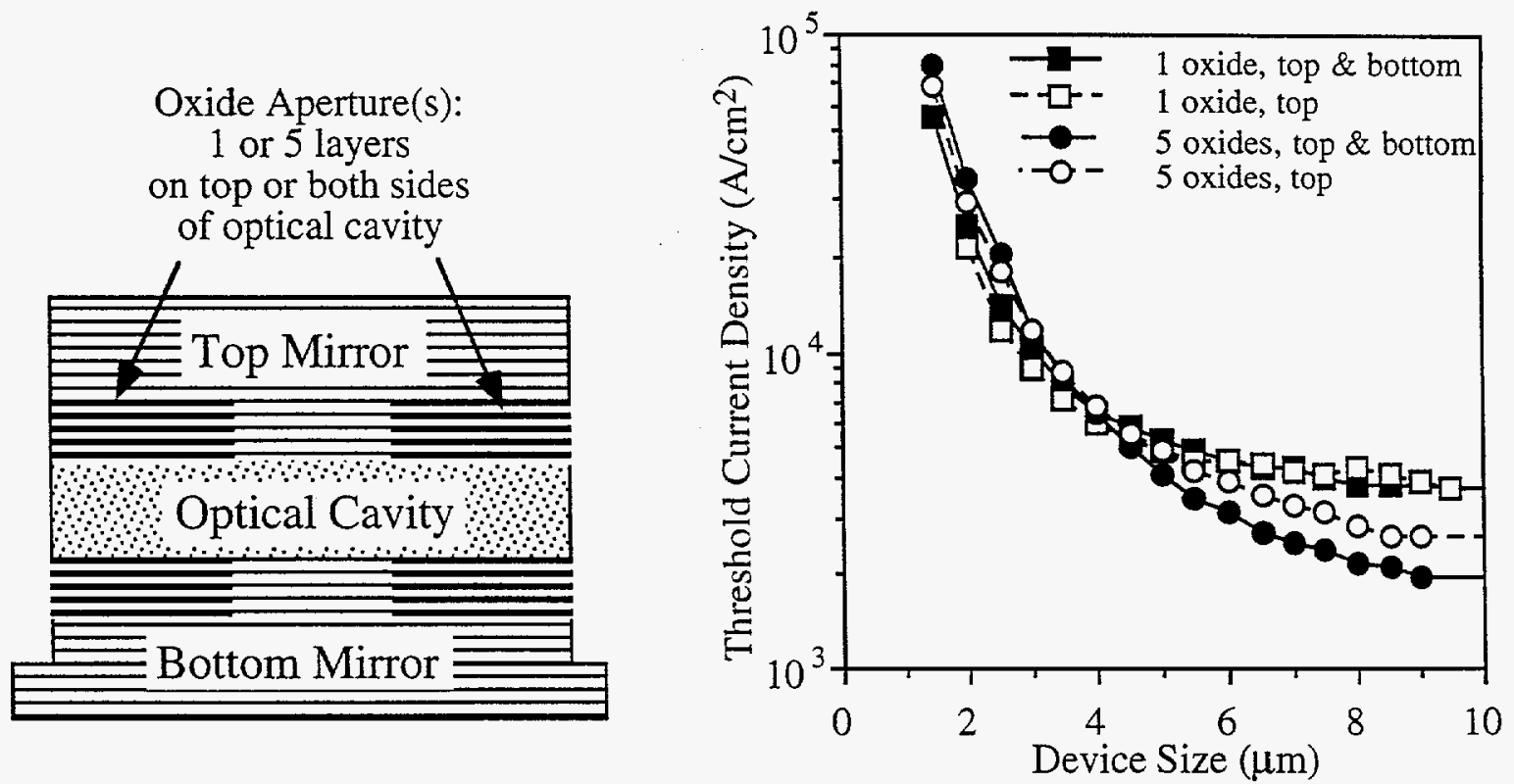

Fig. 1 Sketch of selectively oxidized VCSEL. Fig. $2 \mathrm{~J}_{\text {th }}$ for various aperture configurations.

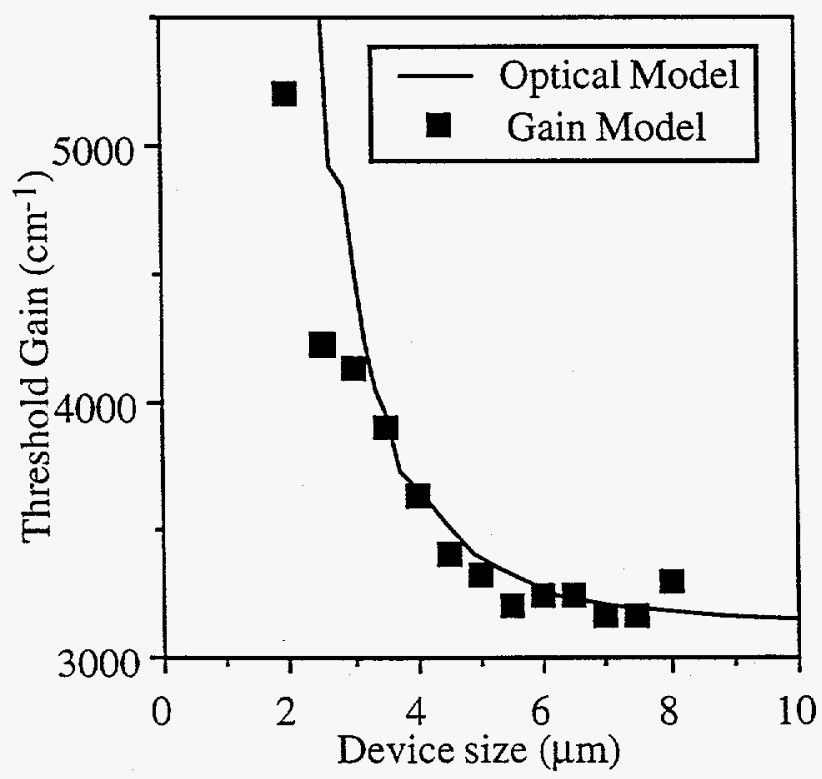

Fig. 3 Material gain for VCSELs with single aperture on each side of cavity.

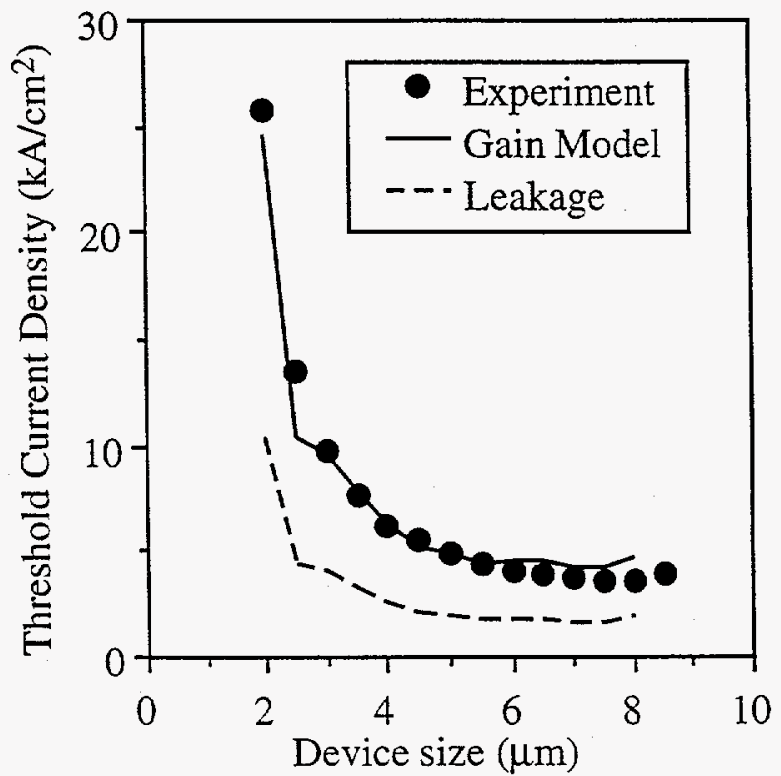

Fig. $4 \mathrm{~J}_{\text {th }}$ and leakage current for VCSELs with single aperture on each side of cavity. 